DE DE GRUYTER
OPEN

\title{
SOME ANALYTIC EXPRESSIONS FOR THE CAPACITANCE AND PROFILES OF THE ELECTRIC DOUBLE LAYER FORMED BY IONS NEAR AN ELECTRODE
}

\author{
DOUGLAS HENDERSON * \\ Department of Chemistry and Biochemistry, Brigham Young University, Provo, Utah 84602, USA

\begin{abstract}
The electric double layer, which is of practical importance, is described. Two theories that yield analytic results, the venerable Poisson-Boltzmann or Gouy-Chapman-Stern theory and the more recent mean spherical approximation, are discussed. The Gouy-Chapman-Stern theory fails to account for the size of the ions nor for correlations amoung the ions. The mean spherical approximation overcomes, to some extent, these deficiencies but is applicable only for small electrode charge. A hybrid description that overcomes some of these problems is presented. While not perfect, it gives results for the differential capacitance that are typical of those of an ionic liquid. In particular, the differential capacitance can pass from having a double hump at low concentrations to a single hump at high concentrations.
\end{abstract}

Keywords: Electric double layer, capacitance, Gouy-Chapman-Stern theory, mean spherical approximation, density functional theory, computer simulation

\section{Introduction}

A double layer (DL) or an electric double layer (EDL) is formed when charged particles are attracted to a charged surface. The most obvious case is an electrolyte near a charged electrode (as in a battery). However, DNA can play a role that is analogous to the electrode. Ions can be attracted to membranes. A membrane can be thought of as a pseudo electrode. Ions are absorbed (often selectively) into physiological channels in membranes. Such channels permit the transport of nutrients into the cell and the removal of waste from the cell and are essential to the functioning of cells and life. The reader's attention is drawn to some recent reviews of EDLs [1-3].

It is the case of an electrolyte near a charged flat surface that is considered here. This is the simplest case; it is an interesting and important application of statistical mechanical theory. The theory of the DL is important to our understanding of batteries. It can be used in the analysis of experimental electrochemical data and in analytical chemistry.

In the model DL that is presented here, the electrode is approximated as a smooth flat charged surface located at $x=0$. This surface is impenetrable and the ions are confined to the region $x>0$. The charge of the electrode is located on the surface. There is no charge inside the electrode $(x<0)$. The electric field does not penetrate the surface. The electrode is a classical metal. Obviously, this is an approximation but there has been very

\footnotetext{
*Author for correspondence: doug@chem.byu.edu
}

little work that takes into account the electronic structure of the electrode. The electrode charge is presumed to be uniform; the charge density of the electrode is $\sigma$ and has the units of $\mathrm{C} / \mathrm{m}^{2}$. Ions in the electrolyte near an electrode that have a charge opposite to that of the electrode are attracted to the electrode and form a layer whose net charge is equal in magnitude, but opposite in electric sign, to the charge of the electrode. The electrode and the attracted charge are together called an EDL. The charge in the EDL of the electrolyte can be spread over an extended region, usually called the diffuse layer, and need not consist solely of counterions whose charge is opposite to the electrode charge. The counterions can bring some coions with them. There may be regions of alternating charge where the coions predominate. However, the net charge of the attracted charged region in the electrolyte is equal in magnitude but opposite in sign to that of the electrode. Otherwise, the electric field would not vanish far from the electrode.

For simplicity, the model electrolyte that is employed here is a fluid of charged hard spheres of diameter $d$. In this study, the electrolyte is assumed to be binary. For additional simplicity, the ions are assumed in this article to be symmetric both in the magnitude of their charge and diameter. The value of the charge of an ion of species $i$ is $z_{i} e$, where $z_{i}$ is the ion valence and has the sign of the ion charge. The magnitude of the elementary charge is $e$. Because the ions are symmetric, $\left|z_{i}\right|=z$. In the bulk, the density of the ions of species $i$ is $\rho_{i}=N_{i} / v$, where $N_{i}$ is the number of ions of species $i$ in the bulk 
and $v$ is the volume of the system. Electrical neutrality requires that $N_{1}=N_{2}$ or $\rho_{1}=\rho_{2}$ or $\sum z_{i} \rho_{i}=0$. The solvent (usually water) of the electrolyte is characterized by a dielectric constant, $\epsilon$. Any change of the dielectric constant with a change of ion concentration is ignored. This model electrolyte is appropriately called the primitive model (PM). In the particular case considered here, where the ions all have the same diameter, this model is called the restricted primitive model (RPM).

In this model, the interaction between a pair of ions, whose centers are separated by the distance $r$, is given by

$$
u_{i j}(r)=\left\{\begin{array}{ll}
\infty & \text { for } \quad r<d \\
\frac{z_{i} z_{j} e^{2}}{4 \pi \epsilon_{0} \epsilon r} & \text { for } \quad r \geq d
\end{array},\right.
$$

where $\epsilon_{0}$ is the permittivity of free space, and the interaction of an ion with the surface is given by

$$
u_{\mathrm{w} i}(x)=\left\{\begin{array}{ll}
\infty & \text { for } \quad x<d / 2 \\
-\frac{\sigma z_{i} e x}{\epsilon_{0} \epsilon} & \text { for } \quad x \geq d / 2
\end{array},\right.
$$

where $x$ is the distance between the center of the ion and the surface.

Our task is to determine the density profile, $\rho_{i}(x)$, of the ions, or equivalently, $g_{i}(x)=\rho_{i}(x) / \rho_{i}$. Note that $\rho_{i}(\infty)=\rho_{i}$, so that $g_{i}(\infty)=1$. Once, the $g_{i}(x)$ are known, the charge profile $\left(\mathrm{C} / \mathrm{m}^{2}\right)$, for $x>d / 2$, is given by

$$
q(x)=e \sum_{i} z_{i} \rho_{i} h_{i}(x)
$$

where $h_{i}(x)=g_{i}(x)-1$. In writing Eq. 3, the global charge neutrality condition $\sum z_{i} \rho_{i}=0$ has been invoked. The charge density on the electrode is given by

$$
\sigma=-e \sum_{i} z_{i} \rho_{i} \int_{d / 2}^{\infty} h_{i}(t) d t
$$

There is no point including the region $0<t<d / 2$ in the integral since $\sum h_{i}(t)=0$ in this region. The potential profile (in Volts) is given by

$$
\phi(x)=-\frac{e}{\epsilon \epsilon_{0}} \sum_{i} z_{i} \rho_{i} \int_{x}^{\infty}(t-x) h_{i}(t) d t .
$$

In particular, the potential (Volts) of the electrode is given by

$$
V=\phi(0)=-\frac{e}{\epsilon \epsilon_{0}} \sum_{i} z_{i} \rho_{i} \int_{0}^{\infty} t h_{i}(t) d t .
$$

Note that these equations satisfy Poisson's equation

$$
\frac{d^{2} \phi(x)}{d x^{2}}=-\frac{q(x)}{\epsilon \epsilon_{0}}
$$

Indeed, Eqs. 3 and 5 are obtained by integrating Poisson's equation. An alternative procedure for computing the potential profile has been proposed by Boda and Gillespie [4] for simulation purposes.
It is often convenient to use dimensionless, or reduced, values that are denoted by an asterisk. For a system whose temperature $(\mathrm{K})$ is $T$, the reduced temperature is $T^{*}=4 \pi \epsilon \epsilon_{0} d k T / z^{2} e^{2}$. The reduced density is $\rho_{i}^{*}=\rho_{i} d^{3}$, the reduced electrode charge density is $\sigma^{*}=\sigma d^{2} / e$, and the reduced potential is $\phi^{*}=\beta e \phi$, where $\beta=1 / k T$, with $k$ being the Boltzmann constant (the gas constant per particle).

\section{Poisson-Boltzmann or Gouy-Chapman-Stern theory: comparison with simulations}

The classic theory of the EDL was developed by Gouy [5], Chapman [6], and Stern [7] (GCS) a century ago. The theory is based on Poisson's equation together with the Boltzmann formula,

$$
g_{i}(x)=\left\{\begin{array}{ll}
0 & x<d / 2 \\
\exp \left[-\beta z_{i} e \phi(x)\right] & x \geq d / 2
\end{array} .\right.
$$

In electrostatics, Poisson's equation is exact and is equivalent to one of Maxwell's equations. The Boltzmann formula is approximate and neglects ion size and correlations between the ions. Eq. 8 states that $g_{i}(x)$ for the coions is the reciprocal of $g_{i}(x)$ for the counterions. This is not true, in general [8].

Equation 8, when inserted into Poisson's equation, yields what may be called the Poisson-Boltzmann (PB) or GCS approximation. This approximation is also employed in the Debye-Hückel (DH) theory for bulk electrolytes that was developed some years later. However, because of the three dimensional geometry of the DH theory, the nonlinear PB equation cannot be solved analytically and the PB equations in the DH theory are usually linearized. In the GCS theory, the resultant PB equation is a nonlinear second order differential equation. As has been pointed out, such equations generally do not yield analytic solutions. However, for the one dimensional geometry of the planar DL that is considered here, an analytic solution is possible in the case of the GCS theory.

The resulting $\mathrm{PB} / \mathrm{GCS}$ potential is

$$
\begin{aligned}
\frac{\beta z e \phi(x)}{2} & =\ln \left\{1+\frac{b / 2}{1+\sqrt{1+b^{2} / 4}} \exp [-\kappa y]\right\} \\
& -\ln \left\{1-\frac{b / 2}{1+\sqrt{1+b^{2} / 4}} \exp [-\kappa y]\right\},
\end{aligned}
$$

where $y=x-d / 2>0$ and

$$
b=\frac{\beta z e \sigma}{\epsilon \epsilon_{0} \kappa}
$$

where $\kappa$ is the Debye screening parameter that is given by

$$
\kappa=\sqrt{\frac{\beta z^{2} e^{2} \rho}{\epsilon \epsilon_{0}}}
$$


with $\rho=\sum \rho_{i}$. The parameter $b$ is another dimensionless measure of the electrode charge density. However, it is not as fundamental a quantity as $\sigma^{*}$ since it arises from a theory. The parameter $\kappa$ is a screening parameter; it is an inverse measure of the distance over which the profiles reach their asymptotic values within the GCS and DH theories.

In the GCS theory the relationship between the potential difference and electrode charge density is given by

$$
\sinh \left[\frac{\beta z e \phi_{d / 2}}{2}\right]=\frac{b}{2},
$$

where $\phi_{d / 2}=\phi(d / 2)$ is often called the diffuse layer potential. Some relations that are equivalent to Eq. 12 are

$$
\begin{aligned}
& \cosh \left[\frac{\beta z e \phi_{d / 2}}{2}\right]=\sqrt{1+b^{2} / 4}, \\
& \tanh \left[\frac{\beta z e \phi_{d / 2}}{2}\right]=\frac{b / 2}{\sqrt{1+b^{2} / 4}},
\end{aligned}
$$

and

$$
\tanh \left[\frac{\beta z e \phi_{d / 2}}{4}\right]=\frac{b / 2}{1+\sqrt{1+b^{2} / 4}} .
$$

The equivalence of Eqs. 12-15 is a result of identities among the hyperbolic functions.

Thus, Eq. 9 can be written as

$$
\begin{aligned}
\frac{\beta z e \phi(x)}{2} & =\ln \left\{1+\tanh \left[\frac{\beta z e \phi_{d / 2}}{4}\right] \exp (-\kappa y)\right\} \\
& -\ln \left\{1-\tanh \left[\frac{\beta z e \phi_{d / 2}}{4}\right] \exp (-\kappa y)\right\} .
\end{aligned}
$$

Alternative forms of Eqs. 9 and 16 are

$$
\tanh \left[\frac{\beta z e \phi(x)}{4}\right]=\tanh \left[\frac{\beta z e \phi_{d / 2}}{4}\right] \exp (-\kappa y)
$$

or

$$
\tanh \left[\frac{\beta z e \phi(x)}{4}\right]=\frac{b / 2}{1+\sqrt{1+b^{2} / 4}} \exp (-\kappa y) .
$$

In the GCS theory, the potential difference across the EDL is

$$
V=-\frac{z e}{\epsilon \epsilon_{0}} \sum_{i} \rho_{i} \int_{0}^{\infty} t h_{i}(t) d t=\frac{\sigma d}{2 \epsilon \epsilon_{0}}+\phi_{d / 2}
$$

where $\phi_{d / 2}$ is given by Eq. 12 . Thus, the capacitance, $C=\sigma / V$, of the EDL is

$$
\frac{1}{C}=\frac{d}{2 \epsilon \epsilon_{0}}+\frac{2 \sinh ^{-1}(b / 2)}{\epsilon \epsilon_{0} \kappa b}
$$

and the differential capacitance, $C_{\mathrm{d}}=\partial \sigma / \partial V$, of the EDL is given by

$$
\frac{1}{C_{\mathrm{d}}}=\frac{d}{2 \epsilon \epsilon_{0}}+\frac{1}{\epsilon \epsilon_{0} \kappa \sqrt{1+b^{2} / 4}} .
$$

Equations 20 and 21 are formally identical to a diffuse layer capacitor with capacitance,

$$
C^{\mathrm{dl}}=\epsilon \epsilon_{0} \kappa \frac{b / 2}{\sinh ^{-1}(b / 2)},
$$

or differential capacitance

$$
C_{\mathrm{d}}^{\mathrm{dl}}=\epsilon \epsilon_{0} \kappa \sqrt{1+b^{2} / 4}
$$

in series with an inner-layer parallel plate capacitor with capacitance (or differential capacitance),

$$
C^{\mathrm{il}}=C_{\mathrm{d}}^{\mathrm{il}}=\frac{2 \epsilon \epsilon_{0}}{d} .
$$

At contact,

$$
g_{i}(d / 2)=\exp \left[\beta z_{i} e \phi_{d / 2}\right]=1+\frac{b^{2}}{2}-\frac{z_{i}}{z} b \sqrt{1+\frac{b^{2}}{4}},
$$

so that $g_{\text {sum }}(d / 2)=\frac{1}{2}\left[g_{1}(d / 2)+g_{2}(d / 2)\right]$ is, in the GCS theory, given by

$$
g_{\mathrm{sum}}(d / 2)=1+\frac{b^{2}}{2} .
$$

This is to be compared with the exact result (for the restricted PM) due to Henderson and Blum [9] and Henderson, Blum, and Lebowitz [10],

$$
g_{\text {sum }}(d / 2)=\frac{p}{\rho k T}+\frac{b^{2}}{2},
$$

where $p$ is the osmotic pressure of the electrolyte. The second term in the above equation is just the Maxwell electrostatic stress. Thus, Eq. 27 is just a force balance condition where the momentum transfer to the electrode is equal to the sum of the osmotic term and the Maxwell stress. The GCS theory deals with the electrostatic term correctly but replaces the osmotic pressure with the ideal gas result $p=\rho k T$ because of the neglect of the ion diameters.

For comparison with the mean spherical approximation (MSA), which is a linear response theory that will be considered in the next section, it is worthwhile to give the linearized GCS theory results, obtained for the case of small electrode charge. In this case,

$$
g_{i}(x)=\left\{\begin{array}{ll}
0 & \text { for } \quad x<d / 2 \\
1-\beta z_{i} e \phi(x) & \text { for } \quad x \geq d / 2
\end{array},\right.
$$

or

$$
g_{i}(x)=\left\{\begin{array}{ll}
0 & \text { for } \quad x<d / 2 \\
1+\frac{\beta z_{i} e \sigma}{\epsilon \epsilon_{0} \kappa} \exp (-\kappa y) & \text { for } x \geq d / 2
\end{array} .\right.
$$

The potential profile in the diffuse layer is given by

$$
\phi(x)=\phi_{d / 2} \exp (-\kappa y)
$$

with $\phi_{d / 2}$ given by

$$
\phi_{d / 2}=\frac{\sigma}{\epsilon \epsilon_{0} \kappa} .
$$




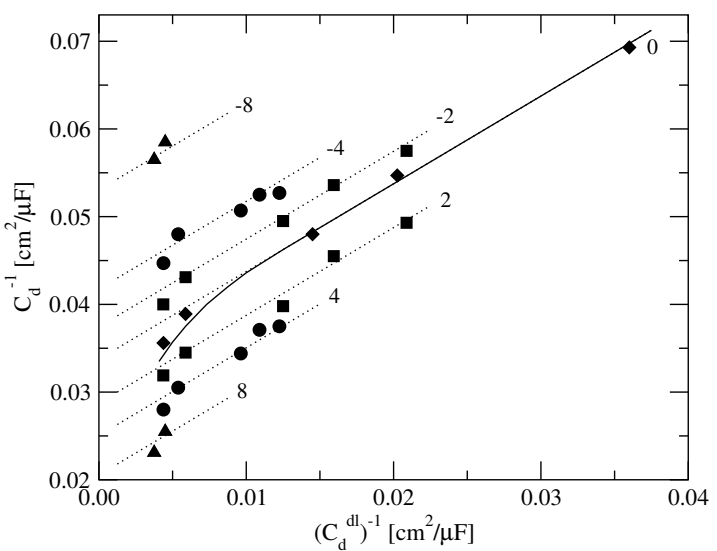

Figure 1: Experimental values of the inverse differential capacitance, $C_{\mathrm{d}}$, of an aqueous solution of $\mathrm{NaH}_{2} \mathrm{PO}_{4}$ at 25 ${ }^{\circ} \mathrm{C}$ near a hanging drop mercury electrode as a function of the inverse diffuse layer capacitance, $C_{\mathrm{d}}^{\mathrm{dl}}$, obtained from Eq. 23. The points are the experimental results of Parsons and Zobel. The light straight lines of unit slope give the results of the GCS theory but with the experimental inner layer capacitance obtained empiricially. The numbers at the low concentration end of the lines give the electrode charges in units of $\mu \mathrm{Ccm}^{-2}$. The heavy solid curve gives the results of the MSA using a dipolar hard sphere model for the solvent together with an estimate of the contribution of the electronic structure of the electrode and is intended only as an aid to the eye. This figure has been reproduced, with permission, from Ref. [1].

The potential difference across the EDL is

$$
V=\frac{\sigma d}{2 \epsilon \epsilon_{0}}+\frac{\sigma}{\epsilon \epsilon_{0} \kappa}
$$

and the capacitance (and differential capacitance) of the EDL is given by

$$
\frac{1}{C}=\frac{d}{2 \epsilon \epsilon_{0}}+\frac{1}{\epsilon \epsilon_{0} \kappa} .
$$

In the GCS theory, in the limit of large $\kappa$ (high concentration) or large $b$ (high electrode charge), the diffuse layer capacitance is large and, as a result, the inner layer capacitance dominates, due to the reciprocal or series additivity of Eqs. 20 and 21. Hence, in the GCS theory, $C=2 \epsilon \epsilon_{0} / d$ is the limiting (maximum) value of $C$ or $C_{\mathrm{d}}$ at large concentrations or large electrode charges. Further, the differential capacitance at low concentrations looks something like a parabola but flattens out at large $\sigma$. At high concentrations, the differential capacitance is constant. Any additional shape in the experimental differential capacitance is added by an empirical fit of the inner layer capacitance to the experimental results. However, the diffuse layer capacitance is presumed to be given adequately by the GCS theory.

Parsons and Zobel (PZ) [11] have plotted their experimental results for the inverse of the differential capacitance as a function of the inverse of the diffuse layer dif- ferential capacitance, given by Eq. 23. Such a plot is often called a Parsons-Zobel plot. If the GCS theory were correct, this should result in a straight line. The extrapolation of the straight line to $1 / C_{\mathrm{d}}^{\mathrm{dl}}=0$ (high concentration and/or high electrode charge density) should, if the GCS theory were correct, yield the reciprocal of the inner layer capacitance. As is seen in Fig. 1, at first sight the experimental results of PZ (the points) do seem to follow a straight line and, conventionally, are presumed to provide an experimental verification of the GCS theory. In Fig. 1 , the light straight lines are the GCS results. The solid curve is the result of the MSA that has not yet been discussed. For the moment the solid line can be considered to be an aid to the eye in following the trend of the experimental results. In the conventional GCS picture, the inner layer capacitance might not be given by Eq. 24 but might differ because of the presumed effect of the presence and nature of the solvent molecules and the electronic structure of the electrode that are beyond the GCS theory. Possible solvent effects might be a lower dielectric constant due to the alignment of the solvent molecules because of the strength of the electrode charge. The important point is that, in the GCS theory, such solvent effects are presumed to be confined only to the inner layer. The GCS theory is conventionally considered to provide an adequate description of the diffuse layer where the ions are present. Also, it is thought to provide a description of the EDL when combined with some treatment of the solvent molecules in the inner layer, or even an empirical fit. Indeed, the extrapolation of the straight lines to $1 / C_{\mathrm{d}}^{\mathrm{dl}}=0$ is one method of obtaining presumed "experimental" values of $C_{\mathrm{d}}^{\mathrm{il}}$.

However, a careful examination of the PZ experimental results in Fig. 1 indicates that the experimental differential capacitance does not follow Eq. 21 at high concentrations (the left side of the figure) but rises above the extrapolated intercept, possibly without limit. Until recently, most experimentalists have ignored this point and did not concern themselves with this issue because ions are not soluble in water when their concentration is large and it is difficult to obtain results with other solvents. Additionally, experimental results are difficult to obtain at high electrode charges for conventional electrolytes. However, as we shall see, DLs in ionic liquids can be formed at high concentrations and the deficiencies of the GCS theory become quite apparent.

Given that experiments on aqueous systems are difficult in regimes where problems with the GCS theory become apparent, it is useful to consider computer simulations. One simulation technique is the Monte Carlo (MC) method. Until recently, it has been the most common simulation tool in DL studies. In MC simulations the ions undergo a random walk and the profiles and other properties of interest are obtained by averages over this random walk. A simple random walk would take forever before useful results could be obtained. However, meaningful results can be obtained by means of a biased random walk that confines the ions to regions in which they 

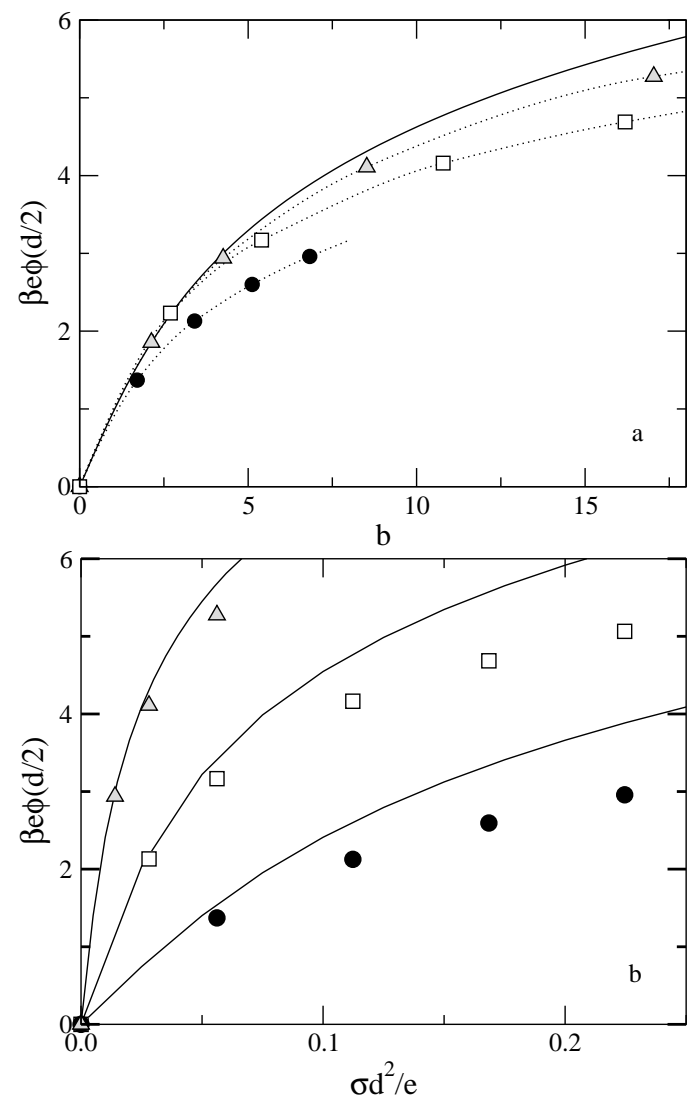

Figure 2: Diffuse layer potential of a 1:1 electrolyte $(d=$ $3 \AA$ ) at room temperature as a function of $b$ (part a) and $\sigma$ (part b). The curves are, from top to bottom, for 0.01 , 0.1 , and $1 \mathrm{M}$ solutions. The symbols give the simulation results. The solid curve in part a gives the GCS results. The dotted lines connect the MC results for easier visualization. The lines in part $\mathrm{b}$ give the GCS results. Part $\mathrm{a}$ is reproduced, with permission, from Ref. [1].

have a high probability of residing. The simulation cell consists of a parallelopiped with a charged wall (the electrode) at $x=0$ and another wall (charged or uncharged) at $x=L$, where $L$ is so large that the two walls do not interfere. Periodic boundary conditions are used in the other two directions. The size of the cell is chosen to be large enough that electrostatic screening eliminates the effects of the periodic image cells. The number of ions of each species is chosen so that the system is electroneutral. The first use of MC simulations for the study of the EDL was that of Torrie and Valleau [12,13]. After their seminal studies, there was a hiatus in simulation studies of the EDL. However in recent years, there has been a renewed interest in simulations of the EDL that includes the work of Bhuiyan et al. [8], Boda et al. [14,15], and Lamperski et al. [16,17].

Another simulation technique is the molecular dynamics (MD) method in which the equations of motion are solved and the properties of the system of interest are obtained by averaging over the positions and velocities of the ions. A simulation cell that is similar to that used

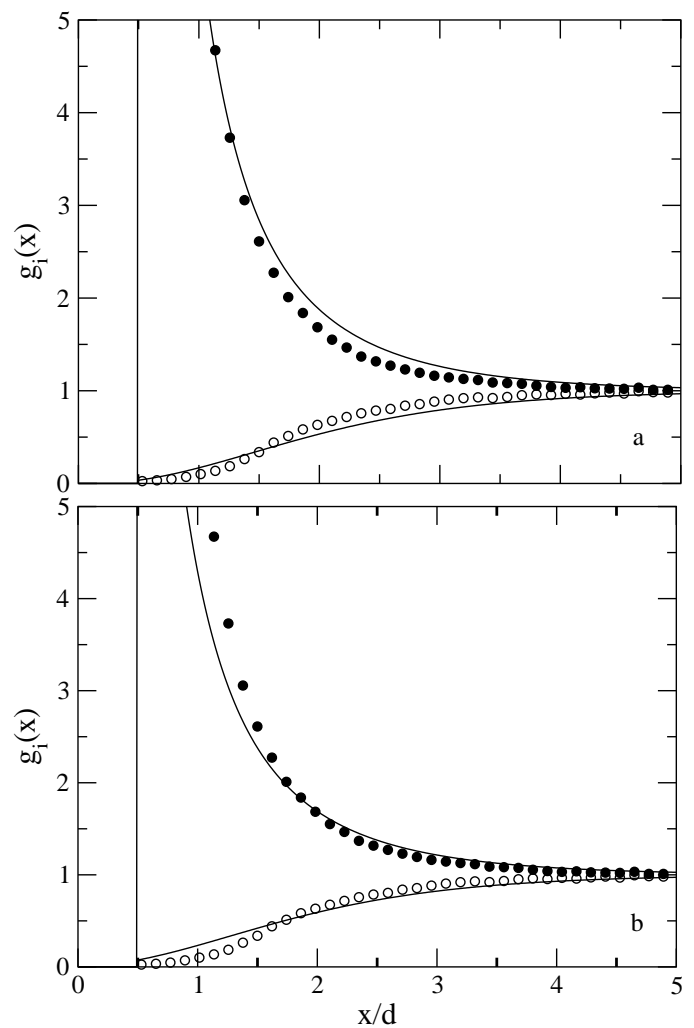

Figure 3: Normalized density profiles, $g_{i}(x)$, of a 1:1 electrolyte $(d=3 \AA)$ at $1 \mathrm{M}$ and room temperature for the state for which the MC values are $\sigma d^{2} / e=0.1685$ and $\beta e \phi(d / 2)=2.6$. The points give the simulation results and the curves give the GCS results. The comparison is made at the same charge density (part a) and the same diffuse layer potential (part b).

in $\mathrm{MC}$ simulations is employed. In recent years, there has been an interest in MD simulations of the EDL, especially for ionic liquids. Some representative studies are those of Vatamanu et al. [18, 19], Hu et al. [20], and Feng [21] et al.

A comparison with simulation gives an unambiguous test of the GCS theory since uncertainties resulting from empirical fits of the diffuse layer capacitance cannot arise. The GCS theory and simulations both use the same model and interaction parameters that are defined in Eqs. 1 and 2. Additionally, simulations and theory give results for the density profiles, $g_{i}(x)$, that cannot be obtained by present experimental methods. The simulations plotted in Figs. 2-5 are those of Boda et al. [22]. In Fig. 2a, the electrostatic potential $\beta e \phi(d / 2)$ for a 1:1 electrolyte is plotted as a function of $b$ for three concentrations $(0.01 \mathrm{M}, 0.1 \mathrm{M}$, and $1 \mathrm{M})$. If the GCS theory were correct, these curves would be identical and independent of concentration. Hence, there can be only one GCS curve in Fig. 2a. As is seen, $\phi(d / 2)$ as a function of $b$ actually decreases with increasing concentration. In Fig. $2 b, \phi(d / 2)$ is plotted as a function of $\sigma d^{2} / e$. The CGS curves are greater than the simulation results, espe- 


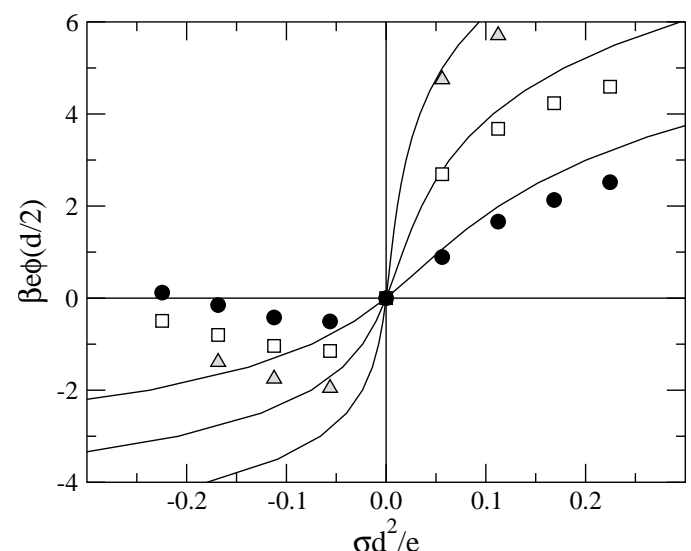

Figure 4: Diffuse layer potential of a 2:1 electrolyte $(d=$ $3 \AA$ ) at room temperature as a function of $\sigma$. The curves are, from top to bottom, for $0.01,0.1$, and $1 \mathrm{M}$ for positive $\sigma$ and the reverse for negative $\sigma$. The symbols give the simulation results. The curves give the GCS results.

cially as the concentration increases. The density profiles for a 1:1 electrolyte are plotted in Fig. 3. The comparison is made at the same value of $\sigma$ in part a and the same value of $\phi(d / 2)$ in part $b$. In principle, there is no reason to choose whether the comparison should be made at the same $\sigma$, the same $\phi(d / 2)$, or the same $\phi(0)$. It was natural for Torrie and Valleau to make their comparisons at the same $\sigma$ because $\sigma$ is the input variable in their method. However, $\phi$ is the natural variable in the GCS theory. In any case, the GCS theory looks best when $\sigma$ is used as the input variable. Torrie and Valleau overstated things when they said that the GCS theory was reasonable for a 1:1 electrolyte. Their statement is most applicable if the comparison is made at the same value of $\sigma$. The value of the counterion profile would be in poor agreement at $x=d / 2$ if $\phi(d / 2)$ or $\phi(0)$ were used as the input variable. A similar comparison is made for a 2:1 electrolyte in Figs. 4 and 5. The agreement of the GCS theory with simulations is much poorer. The electrostatic interactions are stronger because of the presence of the divalent ions. When the divalent ions are the counterions, the potential, $\phi(d / 2)$ has a maximum and then decreases with increasing electrode charge. This is not seen in the GCS results which are monotonic. Further, the simulation profiles are not monotonic whereas the GCS results are monotonic. The simulation profiles have oscillations. The EDL can consist of regions where counterions or coions predominate. When the coions predominate, this phenomenon is known as charge inversion. Of course, the net charge in the diffuse layer is still equal in magnitude, but opposite in sign, to that of the electrode charge. This is required to screen the electrode charge and potential far from the electrode.

Generally, experimentalists have been content to ignore the discrepancies in the results of the GCS theory and state that these differences are unimportant since they occur at high electrode charges or high concentrations

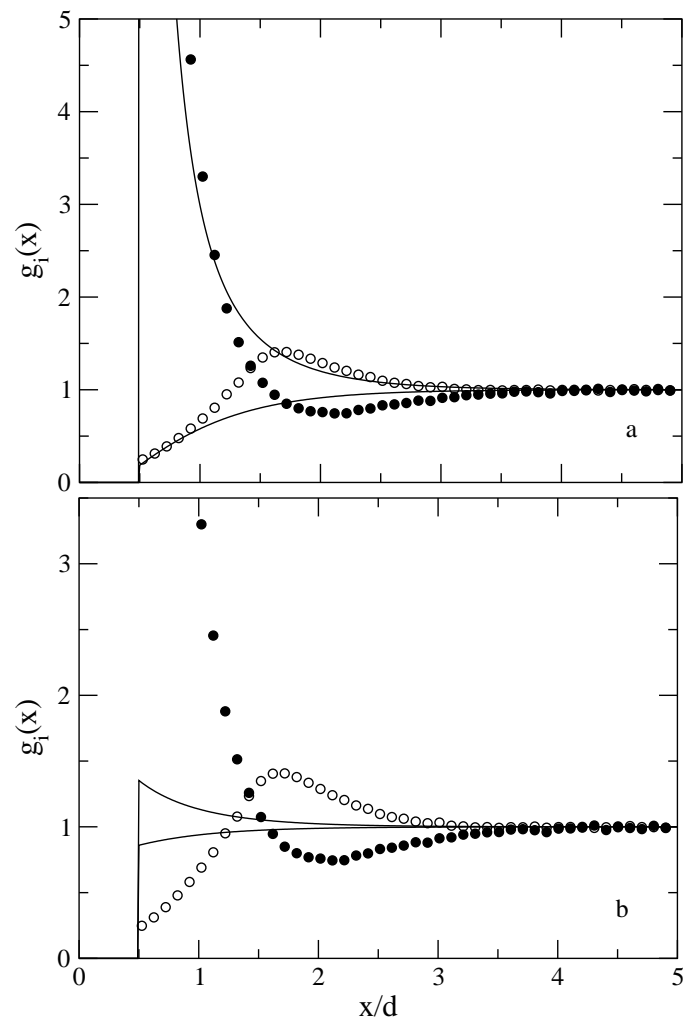

Figure 5: Normalized density profiles, $g_{i}(x)$ of a 2:1 electrolyte $(d=3 \AA)$ at $1 \mathrm{M}$ and room temperature for the state for which the MC values are $\sigma d^{2} / e=-0.1685$ and $\beta e \phi(d / 2)=-0.15$. The points give the simulation results and curves give the GCS results. The comparison is made at the same charge density (part a) and the same diffuse layer potential (part b).

or for high valence electrolytes or nonaqueous systems, where experimental results are difficult to obtain. However, this is short-sighted. As scientists, one of our goals is to understand what is happening. This cannot be done with an inaccurate theory even with curve fitting. In the remainder of this article, attention is directed to more accurate, but still analytic, theories.

\section{Mean spherical approximation}

The mean spherical approximation (MSA) is a natural extension of the linearized GCS theory in which the size of the ions is taken into account. It was first applied to the EDL by Blum [23]. The GCS is usually obtained by means of the solution of a differential equation whereas the MSA is obtained from the solution of an integral equation. At first sight, the connection between the GCS and MSA theories is unclear. However, Henderson and Blum [24] demonstrated that the GCS theory could also be obtained from an integral equation. In fact, the linearized GCS integral equation is just the MSA integral equation with the effect of ion size ignored. Actually, Henderson and Blum proved a more general result. They showed that the GCS theory followed from the 
hypernetted-chain approximation (HNCA) when ion size was neglected. The MSA can be regarded as a linearized version of the HNCA and, because of this, the stated relation of the linearized GCS theory to the MSA follows. In this article, the HNCA is not considered because it does not yield analytic results and has severe problems when $\sigma$ is large [25]. Also, the emphasis in this article is upon analytic, or at least explicit, results that can be valuable in practical calculations.

The MSA result that is analogous to Eq. 29 is

$$
g_{i}(x)= \begin{cases}0 & \text { for } x<d / 2 \\ g_{0}(x)-\frac{\beta z_{i} e \sigma}{\epsilon \epsilon_{0} \kappa} f(y) & \text { for } x \geq d / 2\end{cases}
$$

where $g_{0}(x)$ is the Percus-Yevick (PY) profile for hard spheres near a hard surface. Earlier, Henderson, Abraham, and Barker (HAB) [26] obtained an integral equation for $g_{0}(x)$. The second term gives the electrostatic part of the profile for charged hard spheres near a charged hard surface. Blum [23] did not obtain a result for $f(y)$ but he did obtain an analytic result for the Laplace transform of $f(y)$,

$$
\begin{aligned}
& \int_{0}^{\infty} \exp (-s y) f(y) d y= \\
& \quad=\frac{s}{s^{2}+2(\Gamma \sigma) s+2(\Gamma \sigma)^{2}(1-\exp [-s])},
\end{aligned}
$$

where $2 \Gamma$ is a renormalized screening parameter that is related to $\kappa$ by $\kappa=2 \Gamma(1+\Gamma \sigma)$ or $2 \Gamma \sigma=\sqrt{1+2 \kappa \sigma}$ 1. Note that for small $\kappa$ (small concentrations), $2 \Gamma \sigma=$ $\kappa \sigma-(\kappa \sigma)^{2} / 2+\cdots$. Thus, the MSA screening parameter is smaller than the GCS screening parameter. This suggests that the MSA EDL is wider than that of the GCS theory. This agrees with the simulation results. The notation of Blum has been followed. However, it might have been preferable if he had incorporated the factor of 2 into the definition of $\Gamma$ so that $\Gamma$ became $\kappa$ at low concentrations.

Note that at low concentrations, the Laplace transform of $f(y)$ becomes $1 / s(1+\kappa s)$. This means that

$$
f(y)=\exp (-\kappa y),
$$

in the limit of low concentrations. Also, $g_{0}(x)=1$ in this limit. Thus, at low concentrations, the MSA becomes the GCS theory. Blum did not invert the Laplace transform of $f(y)$. However, he did obtain the contact value of $f(y)$ by examining the Laplace transform of $f(y)$ at large $s$. He showed that $f(0)=1$. Using the earlier result of HAB for $g_{0}(d / 2)$, the contact value of $g_{i}(x)$ is

$$
g_{i}(d / 2)=\frac{1+2 \eta}{(1-\eta)^{2}}-\frac{\beta z_{i} e \sigma}{\epsilon \epsilon_{0}},
$$

where $\eta=\pi \rho d^{3} / 6$. The MSA contact value is an improvement over the GCS result that contains only the ideal gas term. However, the osmotic pressure should have both a hard sphere term and an electrostatic term. Additionally, the hard sphere term is accurate only for low values of $\rho$.
Equation 37 does not contain the quadratic term $b^{2}$ of Eq. 27. This is because the MSA is a linearized theory. A better expression for the osmotic term is

$$
\frac{p}{\rho k T}=\frac{1+\eta+\eta^{2}-\eta^{3}}{(1-\eta)^{3}}-\frac{\Gamma^{3}}{3 \pi \rho},
$$

where $\Gamma$ is the renormalized screening parameter that has been defined above. This result is obtained from the application of the MSA to bulk electrolytes. The MSA, as is the case for most theories, is not fully self-consistent. Henderson et al. [27] have compared this expression with their simulations (see their Fig. 1) and found it to be very accurate. Despite these problems, the MSA contact value given in Eq. 37 does represent an advance.

By expansion of the Laplace transform of $f(y)$, it is easy to show that the MSA EDL satisfies electroneutrality. That is, the charge in the EDL is equal in magnitude, but opposite in sign, to the electrode charge. Again, by expanding the Laplace transform, the MSA expressions for the total and diffuse layer potentials of the EDL are found to be

$$
V=\frac{\sigma}{\epsilon \epsilon_{0}(2 \Gamma)}
$$

and

$$
\phi_{d / 2}=\frac{\sigma}{\epsilon \epsilon_{0} \kappa}\left[1-(\Gamma d)^{2}\right] .
$$

Thus, in the MSA, the capacitance (and differential capacitance) of the EDL is

$$
\frac{1}{C}=\frac{1}{\epsilon \epsilon_{0}(2 \Gamma)}
$$

Expanding the expression that defines $\Gamma$,

$$
2 \Gamma=\kappa-\kappa^{2} d / 2+\kappa^{3} d^{2} / 2+\cdots .
$$

Therefore,

$$
\frac{1}{C}=\frac{1}{\epsilon \epsilon_{0} \kappa}+\frac{d}{2 \epsilon \epsilon_{0}}-\frac{\kappa d^{2}}{4 \epsilon \epsilon_{0}}+\cdots
$$

The MSA capacitance does not reach a maximum at $2 \epsilon \epsilon_{0} / d$ but continues to increase, as is indicated in Fig. 1. The MSA (solid) curve in Fig. 1 was not calculated from Eq. 41 but from a more sophisticated version of the MSA, that is not discussed in detail here, which includes the contribution resulting from explicit solvent molecules and the electronic structure of the metal [28-30]. However, the results of Eq. 41 are qualitatively similar to the more sophisticated results. In this paper, the solid curve serves to guide the eye. The inner layer capacitance continues to be $2 \epsilon \epsilon_{0} / d$ but it is simply the electrode charge divided by potential difference across the inner layer and not a 'catch all' for the deficiencies of the GCS theory. The diffuse capacitance is the electrode charge divided by the potential difference from the distance of closest approach to the bulk electrolyte and contains correction terms to the GCS theory. This is the reverse of the usual interpretation of the GCS theory where the GCS expressions are assumed to be accurate for the diffuse layer 


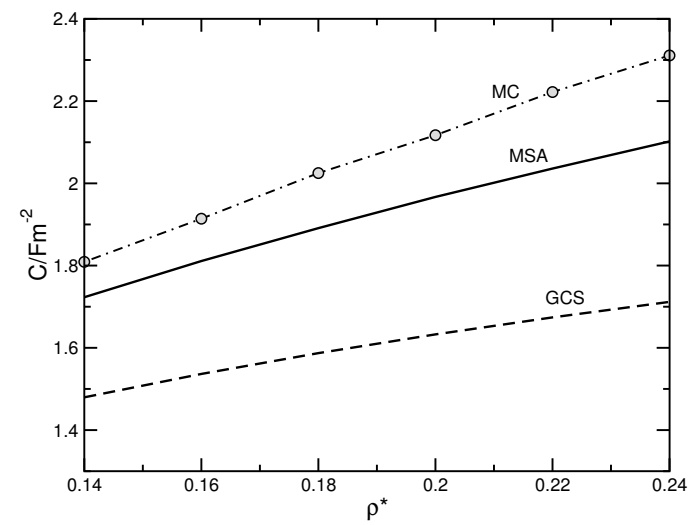

Figure 6: Double layer capacitance, $C$, as a function of the reduced density, $\rho^{*}=\rho d^{3}$, at the reduced temperature $T^{*}=0.08$. The circles are the MC data of Henderson et al. [27] and the lines are the MSA and GCS results. The line through the circles is given as a guide to the eye. Because the MSA is a linearized theory, the MC, MSA, and GCS capacitances are for $\sigma=0$.

capacitance and everything else is 'lumped' into the inner layer capacitance. In the more sophisticated version MSA, the contributions due to the solvent molecules appear in both the diffuse and inner layer potentials. The solvent molecule profile is as diffuse as that of the ions. The effect of the molecular nature of the solvent is not confined to the inner layer.

The initial slope of the $\phi(d / 2)$ vs. $\sigma$ curves in Figs. 2 and 4 is just the inverse of the diffuse portion of the capacitance. It is seen that the initial slope of the MC curves is well described by the GCS theory at low concentrations but increasingly falls below the GCS initial slope (the inverse of the differential capacitance) with increasing concentration as was seen in the experimental results in Fig. 1. Henderson et al. [27] compared the GCS and MSA differential capacitances with their simulation results for a broad range of densities and at a temperature that was meant to be qualitatively representative of an ionic liquid. As is seen in Figs. 6 and 7, the MSA results are considerably improved over the GCS results. The comparison with the MC results is made for a small value of $\sigma$ because the MSA is a linearized theory that is applicable only for small $\sigma$.

Although an analytic expression for $f(y)$ is not available, Henderson and Smith [31] were able to obtain a zonal expansion for $f(y)$. They showed that

$$
f(x)=\sum_{n=1}^{\infty} f_{n}(z) u(z)
$$

where $z=t-n+1, t=x / d, u(z)$ is the Heaviside step function that is zero for $z<0$ and one for $z \geq 0$ and

$$
f_{n}(z)=\exp (-\mu) \frac{\mu^{n}}{(n-1) !}\left[j_{n-2}(\mu)-j_{n-1}(\mu)\right]
$$

with $\mu=(\Gamma d) z$. The function $j_{m}(\mu)$ is the spherical

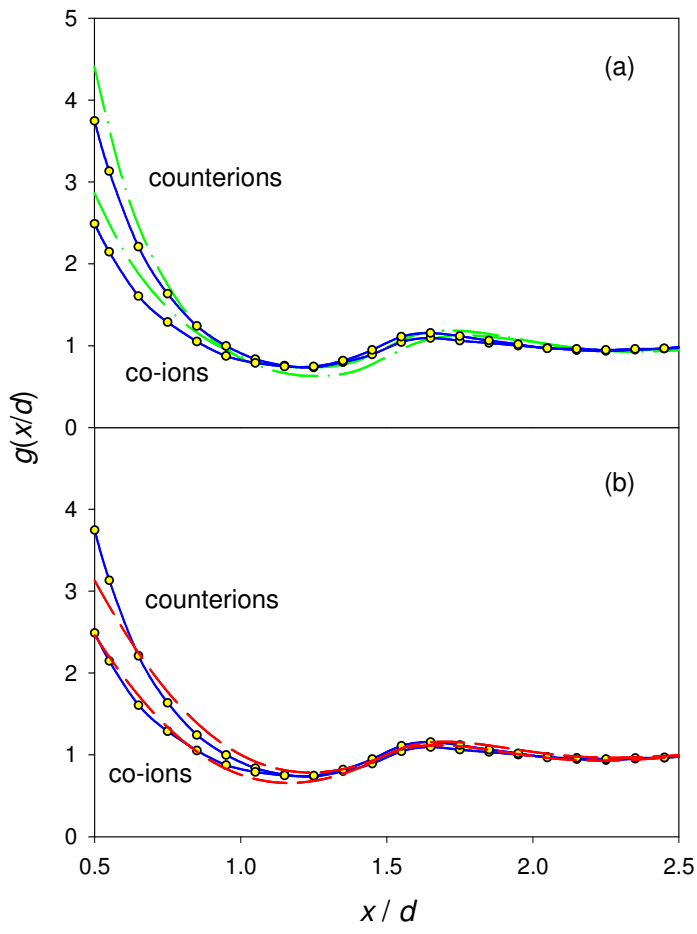

Figure 7: The electrode-ion normalized density profiles, $g_{i}(x / d)$, at the reduced density $\rho^{*}=\rho d^{3}=0.5$ for the reduced temperature $T^{*}=0.8$ and surface charge density, $\sigma=0.05 \mathrm{C} / \mathrm{m}^{2}$, is small enough that the MSA is applicable. The circles are the MC results of Henderson et al. [26] and the dashed line gives the MSA result. The line through the circles is given as a guide to the eye. This figure is reproduced, with permission, from Ref. [26].

Bessel function that is easily calculated using the recurrence formula for this function. Hundreds of $j_{m}(\mu)$ can be calculated without difficulty, even with a laptop computer.

Henderson and Smith [31] also obtained a zonal expansion for $g_{0}(x)$. Their result is

$$
g_{0}(x)=\sum_{n=1}^{\infty} g_{0}^{n}(z) u(z)
$$

where $z$ is again given by $z=t-n+1$ with $t=x / d$. The expressions for the $g_{0}^{n}(x)$ are rather complex. However, Henderson and Smith gave results for $n \leq 5$. The formulae for $g_{0}(x)$ and $f(x)$ are not quite analytic since they involve infinite series. However, these results are explicit and easily used. Fortran programs to obtain $g_{0}(x)$ and $f(x)$ are given in Supplementary Material. Note that the program for $g_{0}(x)$ consists of two parts. One part calculates those parameters that depend only on the state of the electrolyte and the other subroutine in each code calculates profiles for a given $x$. The user should resist the temptation to combine the two parts into one. McQuarrie [32] did this in an appendix to his excellent book and produced an inefficient, and probably incorrect, code that he referred to as 'Henderson's code'. Fortunately, his code 
is illegible in the later printing of his book. If the reader does combine the codes, the reader is on his/her own and should not refer to the combined, or otherwise modified, code as 'Henderson's code'.

The functions $g_{0}(x)$ and $f(x)$ are oscillatory, in accord with the simulations. They are improvements, qualitative and quantitative, to the monotonic functions of the GCS theory.

\section{A useful hybrid description}

As has been mentioned, the deficiencies of the GCS theory could, until recently, be dismissed as appearing mainly under conditions that are of limited experimental interest. However, there has been considerable recent interest in EDLs formed by ionic liquids. Ionic liquids can be thought of as room-temperature molten salts. Because there is no solvent, the ions do not become insoluble in some solvent and experimental results can be obtained at high concentrations. The fact that they exist at room temperature is a great experimental convenience. Kornyshev [33] has drawn attention to these electrochemical systems and aptly suggested that they provide a paradigm change in electrochemistry. He modestly ends the title of his important paper with a question mark. An exclamation mark might have been more appropriate. As well as exposing the deficiencies of the GCS theory, EDLs in ionic liquids are important in green technologies, the design of novel energy storage devices, such as high-tech batteries and super-capacitors [34]. Ionic liquid DLs have attracted recent experimental $[35,36]$ and theoretical interest [18-21, 37-39]. The differential capacitance, as determined by MC simulations, of a simple model [38] of an ionic salt in which $T^{*}=0.8$ and $d=8 \AA$ is given in Fig. 8 for $\rho^{*}=0.04,0.14$ and 0.24 . At low concentrations, the differential capacitance is parabolic-like, as the GCS theory suggests. However, $C_{\mathrm{d}}$ does not become flat at large electrode charges. At higher concentrations, $C_{\mathrm{d}}$ at small electrode charges continues to increase with increasing concentration. This has been seen in Fig. 6 . At high electrode charges, the capacitance decreases. The nature of this decrease seems to be independent of the concentration. This is similar to the GCS theory except that the capacitance is not flat at high electrode charges but decreases. The decrease is due to the fact that the ions are not point charges but occupy space and cannot sit on top of each other. The diffuse layer must become thicker and the capacitance decreases as the electrode charge increases. This is not because the distance of closest approach of the ions increases. Strong secondary peaks in the counterion profile appear [37]. The beginnings of this trend were first observed by Torrie and Valleau [12] and seem to be quite universal.

The GCS theory satisfies Eq. 27 at high electrode charges but fails at low electrode charges whereas the MSA gives reasonable results at small electrode charges. This implies that a repair of the GCS so that it gives the MSA results in the regime of the low electrode charges

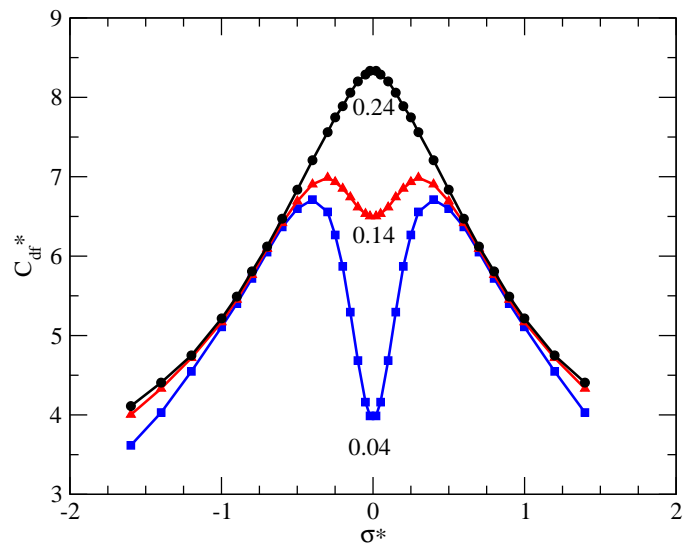

Figure 8: Differential capacitance, $C_{\mathrm{df}}^{*}=C_{\mathrm{d}} d / 4 \pi \epsilon_{0}$, obtained from MC simulation for the EDL of a model ionic liquid with $d=4 \AA$ and $T^{*}=0.8$. The curves are, from bottom to top, for $\rho^{*}=0.04,0.14$, and 0.24 . This figure has been reproduced, with permission, from Ref. [40].

but leaves the high electrode charge part unchanged might be useful. Likely, there is no way to accomplish this in a fundamental way. Additionally, there are probably several semi-empirical ways in which this could be done. Henderson and Lamperski [40] have presented one procedure. Because it is not based on any fundamental ideas, it is not a theory. It would be more appropriate to refer to their procedure as a description. They proposed that the differential capacitance for a symmetric salt could usefully be written as

$$
\frac{1}{C_{\mathrm{d}}}=\frac{d^{\prime}}{2 \epsilon \epsilon_{0}}+\frac{d^{\prime}}{2 \epsilon \epsilon_{0} \sqrt{1+b^{2} / 4}}\left(\frac{1}{\Gamma d^{\prime}}-1\right) .
$$

The parameter $d^{\prime}$ is an adjustable parameter and represents the effective thickness of the diffuse layer. At small $b$ (small electrode charge), Eq. 47 yields

$$
\frac{1}{C_{d}}=\frac{1}{2 \Gamma \epsilon \epsilon_{0}},
$$

which is the MSA result. At large $b$ (large electrode charge), Eq. 47 yields

$$
\frac{1}{C_{d}}=\frac{d^{\prime}}{2 \epsilon \epsilon_{0}} .
$$

The results of Eq. 47, using $d^{\prime}=2 d$ for the system that Lamperski et al. simulated, were given by Henderson and Lamperski. Qualitatively, the results are very similar to the simulation results shown in Fig. 8. Better agreement could be obtained by making $d^{\prime}$ increase with electrode charge. Figure 2 of Henderson and Lamperski suggests that $C_{\mathrm{d}}$ is proportional to $1 / \sigma^{*}$ at large $\sigma^{*}$ (electrode charge) with the proportionality constant being independent of concentration. This behavior was first predicted by Kornyshev [33] on the basis of a lattice theory and seems to be universal. As well as the simulations of Henderson and Lamperski, it has been seen experimentally 


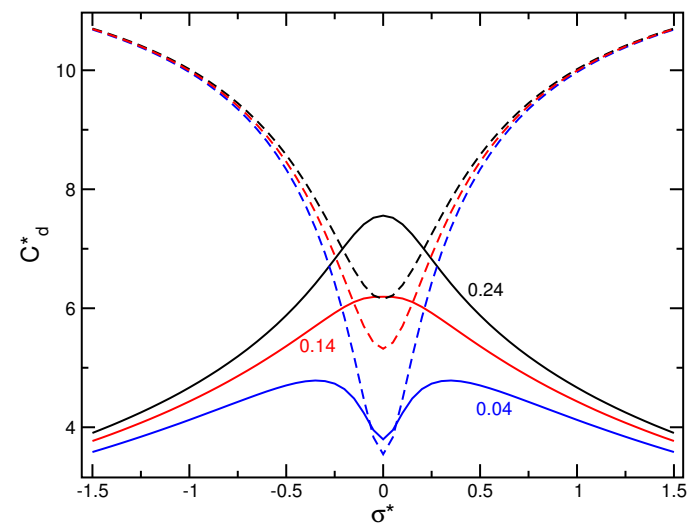

Figure 9: Differential capacitance, $C_{\mathrm{d}}^{*}=C_{\mathrm{d}} d / 4 \pi \epsilon_{0}$, obtained from the hybrid description of the EDL of a model ionic liquid with $d=4 \AA$ and $T^{*}=0.8$. The curves are, from bottom to top, for $\rho^{*}=0.04,0.14$, and 0.24 . The solid and broken curves give the results of the hybrid approach and GCS theory, respectively.

by Islam et al. [35]. Something of the nature of

$$
d^{\prime}=d_{1}+d_{2}\left|\sigma^{*}\right|
$$

would give the desired decrease of $C_{\mathrm{d}}$ at large $\sigma^{*}$. Equation 50 is sensible because it is consistent with the diffuse layer becoming thicker as the electrode charge increases. The results of this ansatz with $d_{1}=2 d$ and $d_{2}=d$ are given in Fig. 9. The results are similar to the simulation results in Fig. 8. This hybrid approach is capable of yielding a capacitance with a double hump at low concentrations and a single hump at high concentrations. This behavior is predicted by simulations and all the good theories of the DL of ionic liquids.

A hybrid treatment of the profiles, $g_{i}(x)$, is possible. One could start with the MSA expression for $g_{i}(x)$ and add to this $g_{i}^{\mathrm{GCS}}(x ; b)-g_{i}^{\mathrm{GCS}}(x ; b=0)$. However, it must be realized that the MSA expressions for the profiles are less accurate than the MSA expressions for the potential and capacitance. The potential and capacitance are integrals and tend to average out any inaccuracies in the profiles.

\section{Conclusion}

The study of the electric DL is an important application of statistical mechanics that is of experimental and applied interest. The GCS theory is popular with experimentalists because it is intuitively simple and easy to use in the routine analysis of experiments. However, the GCS theory has deficiencies. Its use leads to the idea that any problems with the GCS theory can be 'swept under the carpet' by placing all of these problems into an empirical treatment of the inner layer. In reality, the deficiencies of the GCS theory lie with the GCS treatment of the diffuse layer. Admittedly, it is difficult to observe this in aqueous systems. However, it is not impossible.
The departure from linearity in the Parsons-Zobel plot (Fig. 1) is real and should not be ignored. The important field of ionic liquid electrochemistry requires something more adequate than the GCS theory. The best theories of the EDL are the modified Poisson-Boltzmann theory [41] and the density functional theory [42]. However, both theories are numerical and require an iterative numerical solution of a fairly large set of equations and may not be appealing in an experimental analysis. A hybrid description, such as that explored here, preserves the advantage of an analytic treatment of the capacitance and is no more cumbersome than the GCS theory.

\section{Supplementary Information}

Fortran programs to calculate $g_{0}(x)$ and $f(x-d / 2)$ using MSA. The codes can be downloaded free of charge from http://tinyurl.com/hjic-2015-0010-suppl.

\section{Acknowledgement}

Professor Dezsô Boda assisted with the preparation of this paper. Professor Lutful Bari Bhuiyan read the manuscript prior to submission and suggested several important modifications. The author is grateful to both colleagues for their continuing wise advice.

\section{REFERENCES}

[1] Henderson, D., Boda, D.: Insights from theory and simulation on the electrical double layer, Phys. Chem. Chem. Phys., 2009 11(20), 3822-3830 $10.1039 / \mathrm{b} 815946 \mathrm{~g}$

[2] Cherstvy, A.G.: Electrostatic interactions in biological DNA-related systems, Phys. Chem. Chem. Phys., 2011 13, 9942-9968 10.1039/C0CP02796K

[3] Merlet, C., Rotenberg, B., Madden, P.A., Salanne, M.: Computer simulations of ionic liquids at electrochemical interfaces, Phys. Chem. Chem. Phys., 2013 15, 15781-15792 10.1039/c3cp52088a

[4] Boda, D., Gillespie, D.: Calculating the electrostatic potential profiles of double layers from simulation ion density profiles, Hung. J. Ind. Chem., 2013 41(2), 125-132 ISSN: 0133-0276

[5] Gouy, G.: Sur la constitution de la charge electrique a la surface d'un electrolyte, J. de Phys., 1910 9(1), 457-468 10.1051/jphystap:019100090045700

[6] Chapman, D.L.: A contribution to the theory of electrocapillarity, Phil. Mag. Ser. 6, 1913 25(148), 475-481 10.1080/14786440408634187

[7] Stern, O.: Zur Theorie der elektrolytischen Doppelschicht, Zeit. Elektrochem., 1924 30(21-22), 508-516

[8] Bhuiyan, L.B., Outhwaite, C.W., Henderson, D.: Some simulation and modified Poisson-Boltzmann theory results for the contact values of an electrolyte near a charged electrode, J. Electroanal. Chem., 2007 607(1-2), 54-60 10.1016/j.jelechem.2006.10.010 
[9] Henderson, D., Blum, L.: Some exact results and the application of the mean spherical approximation to charged hard spheres near a charged hard wall, J. Chem. Phys., 1978 69(12), 5441-5449 10.1063/1.436535

[10] Henderson, D., Blum, L., Lebowitz, J.L.: Exact formula for the contact value of the density profile of a system of charged hard-spheres near a charged wall, J. Electroanal. Chem., 1979 102(3), 315-319 10.1016/S0022-0728(79)80459-3

[11] Parsons, R., Zobel, F.: The interphase between mercury and aqueous sodium dihydrogen phosphate, J. Electroanal. Chem., 1965 9(5-6), 333-348 10.1016/0022-0728(65)85029-X

[12] Torrie, G.M., Valleau, J.P.: Electrical double-layers 1. Monte Carlo study of a uniformly charged surface, J. Chem. Phys., 1980 73(11), 5807-5816 10.1063/1.440065

[13] Torrie, G.M., Valleau, J.P.: Electrical doublelayers 4. Limitations of the Gouy-Chapman theory, J. Phys. Chem., 1982 86(16), 3251-3257 10.1021/j100213a035

[14] Boda, D., Henderson, D., Chan, K.Y.: Monte Carlo study of the capacitance of the double layer in a model molten salt, J. Chem. Phys., 1999 110(11), 5346-5350 10.1063/1.478429

[15] Boda, D., Henderson, D., Chan, K.Y., Wasan, D.T.: Low temperature anomalies in the properties of the electrochemical interface, Chem. Phys. Lett., 1999 308(5-6), 473-478 10.1016/S0009-2614(99)00643-0

[16] Lamperski, S., Outhwaite, C.W.: Exclusion volume term in the inhomogeneous Poisson-Boltzmann theory for high surface charge, Langmuir, 2002 18(9), 3423-3424 10.1021/la011852v

[17] Lamperski, S., Bhuiyan, L.B.: Counterion layering at high surface charge in an electric double layer. Effect of local concentration approximation, J. Electroanal. Chem., 2003 540, 79-87 10.1016/S0022-0728(02)01278-0

[18] Vatamanu, J., Borodin, O., Smith, G.D.: Molecular insights into the potential and temperature dependences of the differential capacitance of a roomtemperature ionic liquid at graphite electrodes, J. Am. Chem. Soc., 2010 132(42), 14825-14833 10.1021/ja104273r

[19] Vatamanu, J., Borodin, O., Bedrov, D., Smith, G.D.: Molecular dynamics simulation study of the interfacial structure and differential capacitance of Alkylimidazolium Bis(trifluoromethanesulfonyl)imide [Cnmim][TFSI] ionic liquids at graphite electrodes, J. Phys. Chem. C, 2012 116(14), 7940-7951 10.1021/jp301399b

[20] Hu, Z., Vatamanu, J., Borodin, O., Bedrov, D.: A molecular dynamics simulation study of the electric double layer and capacitance of [BMIM][PF6] and [BMIM][BF4] room temperature ionic liquids near charged surfaces, Phys. Chem. Chem. Phys., 2013 15(34), 14234-14247 10.1039/c3cp51218e
[21] Feng, G., Jiang, D., Cummings, P.T.: Curvature effect on the capacitance of electric double layers at ionic liquid/onion-like carbon interfaces, J. Chem. Theor. Comp., 2012 8(3), 1058-1063 $10.1021 / \mathrm{ct} 200914 \mathrm{j}$

[22] Boda, D., Fawcett, W.R., Henderson, D., Sokołowski, S.: Monte Carlo, density functional theory, and Poisson-Boltzmann theory study of the structure of an electrolyte near an electrode, J. Chem. Phys., 2002 116(16), 7170-7176 10.1063/1.1464826

[23] Blum, L.: Theory of electrified interfaces, J. Phys. Chem., 1977 81(2), 136-147 10.1021/j100517a009

[24] Henderson, D., Blum, L.: The Gouy-Chapman theory as a special case of the hypernetted chain approximation, J. Electroanal. Chem., 1978 93(2), 151-154 10.1016/S0022-0728(78)80228-9

[25] Woelki, S., Henderson, D.: Application of the SRISM approach to the study of the capacitance of the double layer of a high density primitive model electrolyte, Cond. Matt. Phys., 2011 14(4), 43801 10.5488/CMP.14.43801

[26] Henderson, D., Abraham, F.F., Barker, J.A.: The Ornstein-Zernike equation for a fluid in contact with a surface, Mol. Phys., 1976 31(4), 1291-1295 10.1080/00268977600101021

[27] Henderson, D., Lamperski, S., Outhwaite, C.W., Bhuiyan, L.B.: A mean spherical approximation study of the capacitance of an electric double layer formed by a high density electrolyte, Coll. Czechoslovak Chem. Comm., 2010 75(3), 303-312 10.1135/cccc2009094

[28] Carnie, S.L., Chan, D.Y.C.: The structure of electrolytes at charged surfaces: Ion-dipole mixtures, J. Chem. Phys., 1980 73(6), 2949-2957 10.1063/1.440468

[29] Blum, L., Henderson, D.: Mixtures of hard ions and dipoles against a charged wall - the OrnsteinZernike equation, some exact results, and the mean spherical approximation, J. Chem. Phys., 1981 74(3), 1902-1910 10.1063/1.441282

[30] Schmickler, W., Henderson, D.: The interphase between jellium and a hard sphere electrolyte. A model for the electric double layer, J. Chem. Phys., 1984 80(7), 3381-3386 10.1063/1.447092

[31] Henderson, D., Smith, W.R.: Exact analytical formulas for the distribution functions of charged hard spheres in the mean spherical approximation, $J$. Stat. Phys., 1978 19(2), 191-200 10.1007/BF01012511

[32] McQuarrie, D.A.: Statistical mechanics (University Science Books, Mill Valley), 2000 ISBN-13: 9781891389153

[33] Kornyshev, A.A.: Double-Layer in Ionic Liquids: Paradigm Change?, J. Phys. Chem. B, 2007 111(20), 5545-5557 10.1021/jp067857o

[34] Winter, M., Brodd, R.J.: What Are Batteries, Fuel Cells, and Supercapacitors?, Chem. Rev., 2004 104(10), 4245-4270 10.1021/cr020730k 
[35] Islam, M.M., Alam, M.T., Ohsaka, T.: Electrical double-layer structure in ionic liquids: A corroboration of the theoretical model by experimental results, J. Phys. Chem. C, 2008 112(42), 1656816574 10.1021/jp8058849

[36] Lockett, V., Horne, M., Sedev, R., Rodopoulos, T., Ralston, J.: Differential capacitance of the double layer at the electrode/ionic liquids interface, Phys. Chem. Chem. Phys., 2010 12(39), 12499-12512 10.1039/C0CP00170H

[37] Wu, J., Jiang, T., Jiang, D., Jin, Z., Henderson, D.: A classical density functional theory for interfacial layering of ionic liquids, Soft Matter, 2011 7(23), 11222-11231 10.1039/c1sm06089a

[38] Lamperski, S., Henderson, D.: Simulation study of capacitance of the electrical double layer of an electrolyte near a highly charged electrode, Mol. Sim., 2011 37(4), 264-268 10.1080/08927022.2010.501973
[39] Lamperski, S., Sosnowska, J., Bhuiyan, L.B., Henderson, D.: Size asymmetric hard spheres as a convenient model for the capacitance of the electrical double layer of an ionic liquid, J. Chem. Phys., 2014 140(1), 014704 10.1063/1.4851456

[40] Henderson, D., Lamperski, S.: Simple Description of the Capacitance of the Double Layer of a High Concentration Electrolyte, J. Chem. Eng. Data, 2011 56(4), 1204-1208 10.1021/je101106z

[41] Outhwaite, C.W., Bhuiyan, L.B.: An improved modified Poisson-Boltzmann equation in electricdouble-layer theory, J. Chem. Soc. Faraday. Trans. II., 1983 79, 707-718 10.1039/F29837900707

[42] Jiang, J., Cao, D., Henderson, D., Wu, J.: A contactcorrected density functional theory for electrolytes at an interface, Phys. Chem. Chem. Phys., 2014 16(9), 3934-3938 10.1039/C3CP55130J 